\title{
Algebraic independence results on the generating Lambert series of the powers of a fixed integer
}

\author{
Peter Bundschuh and Keijo Väänänen
}

\begin{abstract}
In this paper, the algebraic independence of values of the function $G_{d}(z):=\sum_{h>0} z^{d^{h}} /\left(1-z^{d^{h}}\right), d>1$ a fixed integer, at non-zero algebraic points in the unit disk is studied. Whereas the case of multiplicatively independent points has been resolved some time ago, a particularly interesting case of multiplicatively dependent points is considered here, and similar results are obtained for more general functions. The main tool is Mahler's method reducing the investigation of the algebraic independence of numbers (over $\mathbb{Q}$ ) to the one of functions (over the rational function field) if these satisfy certain types of functional equations. Keywords. Algebraic independence of numbers, Mahler's method, algebraic independence of functions.
\end{abstract}

2010 Mathematics Subject Classification. 11J91; 11J81, 39B32

\section{Introduction and main results}

A series of type

$$
\sum_{k=1}^{\infty} \lambda_{k} \frac{z^{k}}{1-z^{k}}
$$

with $\left(\lambda_{k}\right) \in \mathbb{C}^{\mathbb{N}}$ is called a Lambert series. Denoting by $d$ the 'integer' in the title, supposing always $d \geq 2$, and taking $\lambda_{k}$ to be 1 or 0 depending on whether $k$ is a power of $d$ or not, our above series reduces to

$$
G_{d}(z):=\sum_{h=0}^{\infty} \frac{z^{d^{h}}}{1-z^{d^{h}}} .
$$

This series converges exactly on the open unit disk $\mathbb{D}$ and defines there a holomorphic function.

The similar-looking series

$$
F_{d}(z):=\sum_{h=0}^{\infty} \frac{z^{d^{h}}}{1+z^{d^{h}}}
$$

has the same analytic properties and, indeed, we have $F_{d}(z)=-G_{d}(-z)$ in $\mathbb{D}$ if $d$ is odd. Thus, in this case, both functions $F_{d}, G_{d}$ are very closely related.

The aim of the present paper is to study the algebraic independence of the values of the functions $G_{d}(z)$ and $F_{d}(z)$ at certain multiplicatively dependent points $\alpha_{1}, \ldots, \alpha_{n} \in \mathbb{D}$. The arithmetical nature of the values of these functions, being typical examples of Mahler functions, has been studied in several works (see [BV15c], [Coo12], [Coo13], [Mah69], [Sch67]). In particular, it is known that $G_{d}\left(\alpha_{1}\right), \ldots, G_{d}\left(\alpha_{n}\right)$ are algebraically independent (over $\mathbb{Q}$ ) if $\alpha_{1}, \ldots, \alpha_{n} \in \overline{\mathbb{Q}}^{\times} \cap \mathbb{D}$ are multiplicatively independent, and the same holds for $F_{d}$ instead of $G_{d}$. Here and in the sequel, $\overline{\mathbb{Q}}$ denotes the field of all complex algebraic numbers.

In this work, we suppose always

$$
\alpha_{i}:=\alpha^{m_{i}} \quad(i=1, \ldots, n)
$$

with $\alpha \in \overline{\mathbb{Q}}^{\times} \cap \mathbb{D}$ and all $m_{i} \in \mathbb{N}$. The following result was established in [BV15c, Theorem 3.1].

We thank episciences.org for providing open access hosting of the electronic journal Hardy-Ramanujan Journal 
Theorem 1. Let $m_{1}, \ldots, m_{n}$ be $n \geq 2$ positive integers, and let $\alpha \in \overline{\mathbb{Q}}^{\times} \cap \mathbb{D}$. Then $G_{d}\left(\alpha_{1}\right), \ldots, G_{d}\left(\alpha_{n}\right)$ are algebraically independent if and only if

$$
\frac{m_{j}}{m_{i}} \notin d^{\mathbb{Z}}
$$

holds for any pair $(i, j)$ with $i \neq j$.

To state our first new result, we define, for any function $f$, the notation $f( \pm \beta)$ to mean either $f(\beta)$ or $f(-\beta)$.

Theorem 2. Let $m_{1}, \ldots, m_{n}$ be $n \geq 2$ positive integers satisfying the condition (1.3), and let $\alpha \in \overline{\mathbb{Q}}^{\times} \cap \mathbb{D}$. Then, for each choice of $n$ signs, the values $G_{d}\left( \pm \alpha_{1}\right), \ldots, G_{d}\left( \pm \alpha_{n}\right)$ are algebraically independent, and the same holds for $F_{d}\left( \pm \alpha_{1}\right), \ldots, F_{d}\left( \pm \alpha_{n}\right)$.

Note that, as an immediate corollary of this result, we obtain the analogue of Theorem 1 for $F_{d}$.

To state our next result, we define

$$
\mathcal{G}_{d}(z):=\sum_{h=0}^{\infty} \frac{a_{h} z^{d^{h}}}{1-z^{d^{h}}}, \quad \mathcal{F}_{d}(z):=\sum_{h=0}^{\infty} \frac{b_{h} z^{d^{h}}}{1+z^{d^{h}}},
$$

where $\left(a_{h}\right),\left(b_{h}\right)$ are non-zero periodic sequences of algebraic numbers.

Theorem 3. Let $m_{1}, \ldots, m_{n}$ be $n \geq 2$ positive integers such that

$$
\frac{m_{j}}{m_{i}} \notin \mathbb{N}
$$

holds for any pair $(i, j)$ with $i \neq j$, and let $\alpha \in \overline{\mathbb{Q}}^{\times} \cap \mathbb{D}$. Then, for each choice of $n$ signs, the values $\mathcal{G}_{r}\left( \pm \alpha_{i}\right)(i=1, \ldots, n ; r \in \mathbb{N} \backslash\{1\})$ are algebraically independent. In particular, the numbers $G_{r}\left( \pm \alpha_{i}\right)$ $(i=1, \ldots, n ; r \in \mathbb{N} \backslash\{1\})$ are algebraically independent. The same holds if $\mathcal{G}_{r}, G_{r}$ are replaced by $\mathcal{F}_{r}, F_{r}$.

In the remaining results, both functions $G_{d}, F_{d}$ are studied simultaneously. But here the case $d=2$ has to be excluded in a natural way since, using (1.1) and (1.2), we find for $z \in \mathbb{D}$

$$
G_{2}(z)+F_{2}(z)=2 \sum_{h=0}^{\infty} \frac{z^{2^{h}}}{1-z^{2^{h+1}}}=2 \sum_{h=0}^{\infty} \sum_{k=0}^{\infty} z^{(1+2 k) 2^{h}}=2 \sum_{n=1}^{\infty} z^{n}=\frac{2 z}{1-z} .
$$

Theorem 4. Suppose $d \geq 3$, and let $m_{1}, \ldots, m_{n}$ be $n \geq 2$ positive integers satisfying (1.3) and

$$
\frac{m_{j}}{m_{i}} \notin 2 d^{\mathbb{Z}}
$$

for any pair $(i, j)$ with $i \neq j$. If $\alpha \in \overline{\mathbb{Q}}^{\times} \cap \mathbb{D}$, then the numbers $G_{d}\left(\alpha_{1}\right), \ldots, G_{d}\left(\alpha_{n}\right), F_{d}\left(\alpha_{1}\right), \ldots, F_{d}\left(\alpha_{n}\right)$ are algebraically independent.

It may be of some interest to see a typical example of an application of Theorem 4 involving reciprocal sums of the usual Fibonacci numbers $\Phi_{n}$ (or Lucas numbers $\Lambda_{n}$, respectively). To this purpose, we first deduce from (1.1) and (1.2) that $G_{d}\left(\beta^{m}\right)+F_{d}\left(\beta^{m}\right)$, up to an algebraic summand, 
equals to $(2 / \sqrt{5}) \sum_{h>0} 1 / \Phi_{m d^{h}}$ for any $d, m \in \mathbb{N}$ with $2 \mid d$, where we put $\beta:=(1-\sqrt{5}) / 2$. Assuming even $4 \mid d$, Theorem 4 tells us the algebraic independence of all numbers

$$
\sum_{h \geq 0} \frac{1}{\Phi_{m d^{h}}} \quad(m \in 2 \mathbb{N}-1) .
$$

Similarly we establish, for odd $d>2$, the algebraic independence of all $\sum_{h \geq 0} \frac{1}{\Lambda_{m d^{h}}}$ with odd $m$ not divisible by $d$.

Theorem 5. Let $m_{1}, \ldots, m_{n}$ be $n \geq 2$ positive integers such that

$$
\frac{2 m_{j}}{m_{i}} \notin \mathbb{N}
$$

holds for any pair $(i, j)$ with $i \neq j$. If $\alpha \in \overline{\mathbb{Q}}^{\times} \cap \mathbb{D}$, then the numbers $\mathcal{G}_{r}\left(\alpha_{i}\right), \mathcal{F}_{r}\left(\alpha_{i}\right)(i=1, \ldots, n ; r \in$ $\left.\mathbb{N} \backslash\{1\}, r \notin 2^{2 \mathbb{N}-1}\right)$ are algebraically independent. In particular, the numbers $G_{r}\left(\alpha_{i}\right), F_{r}\left(\alpha_{i}\right)(i=$ $1, \ldots, n ; r \in \mathbb{N} \backslash\{1\}, r \notin 2^{2 \mathbb{N}-1}$ ) are algebraically independent.

Remark. Assuming that $\left(d_{h}\right) \in \mathbb{N}^{\mathbb{N}_{0}}$ satisfies a linear recurrence $d_{h+t}=c_{1} d_{h+t-1}+\ldots+c_{t} d_{h}$ with certain conditions on $t$ and $\left(c_{1}, \ldots, c_{t}\right) \in \mathbb{N}_{0}^{t} \backslash\{\underline{0}\}$ excluding, in particular, the case of $\left(d_{h}\right)$ being a geometric progression, Tanaka [Tan05] settled the algebraic independence problem for the values of the Lambert series $\sum_{h \geq 0} z^{d_{h}} /\left(1-z^{d_{h}}\right)$ at distinct points $\alpha_{1}, \ldots, \alpha_{n} \in \overline{\mathbb{Q}}^{\times} \cap \mathbb{D}$. Thus, our investigations on the Lambert series $G_{d}(z)$ just concern the important remaining case, where $\left(d_{h}\right)$ reduces to the geometric progression $\left(d^{h}\right)$.

\section{The main lemma}

The main tool in the proof of [BV15c, Theorem 3.1] was the following auxiliary result.

Lemma 1. Let $m_{1}, \ldots, m_{n}$ be $n \geq 2$ positive integers satisfying condition (1.3). Then the functions $G_{d}\left(z^{m_{1}}\right), \ldots, G_{d}\left(z^{m_{n}}\right)$ are linearly independent over $\mathbb{C}$ modulo $\mathbb{C}(z)$.

Combining the proof of this lemma with some new ideas, we are now able to generalize Lemma 1. To state this generalization, we introduce, for fixed $a \in \mathbb{C}^{\times}$, the functions

$$
G_{d}(a, z):=\sum_{h=0}^{\infty} \frac{a^{h} z^{d^{h}}}{1-z^{d^{h}}}, \quad F_{d}(a, z):=\sum_{h=0}^{\infty} \frac{a^{h} z^{d^{h}}}{1+z^{d^{h}}} .
$$

Lemma 2. Let $m_{1}, \ldots, m_{n}$ be $n \geq 2$ positive integers satisfying condition (1.3). Assume that $I_{1}$ and $I_{2}$ are (possibly empty) disjoint sets of positive integers satisfying $I_{1} \cup I_{2}=\{1, \ldots, n\}$. Then, for any root of unity $\zeta$, the functions $G_{d}\left(\zeta, z^{m_{i}}\right)\left(i \in I_{1}\right), F_{d}\left(\zeta, z^{m_{i}}\right)\left(i \in I_{2}\right)$ are linearly independent over $\mathbb{C}$ modulo $\mathbb{C}(z)$.

Proof. We first note that the functions

$$
g_{i}(z):=G_{d}\left(\zeta, z^{m_{i}}\right), f_{i}(z):=F_{d}\left(\zeta, z^{m_{i}}\right) \quad(i=1, \ldots, n)
$$

satisfy the functional equations

$$
\zeta g_{i}\left(z^{d}\right)=g_{i}(z)+\frac{z^{m_{i}}}{z^{m_{i}}-1}, \zeta f_{i}\left(z^{d}\right)=f_{i}(z)-\frac{z^{m_{i}}}{z^{m_{i}}+1} \quad(i=1, \ldots, n) .
$$


Assume now, contrary to Lemma 2, that there exists some $\underline{c}:=\left(c_{1}, \ldots, c_{n}\right) \in \mathbb{C}^{n} \backslash\{\underline{0}\}$ such that

$$
r(z):=\sum_{i \in I_{1}} c_{i} g_{i}(z)+\sum_{i \in I_{2}} c_{i} f_{i}(z)
$$

is a rational function. For $i \in\{1, \ldots, n\}$, we now write $m_{i}=d^{t(i)} k_{i}$ with integers $t(i) \geq 0, k_{i}>0$ such that $d \nmid k_{i}$. Then condition (1.3) is equivalent to the distinctness of $k_{1}, \ldots, k_{n}$. By using a suitable permutation of $\{1, \ldots, n\}$, we may assume without loss of generality that, for some $m \in\{1, \ldots, n\}$, the conditions $c_{1} \cdots c_{m} \neq 0, c_{m+1}=\cdots=c_{n}=0$ hold and, moreover, $k_{1}>\cdots>k_{m}$. If, under this permutation, $\left\{i \in I_{j}: c_{i} \neq 0\right\}$ changes to $J_{j}(j=1,2)$, then $J_{1} \cup J_{2}=\{1, \ldots, m\}$ and we may write

$$
r(z):=\sum_{i \in J_{1}} c_{i} g_{i}(z)+\sum_{i \in J_{2}} c_{i} f_{i}(z) .
$$

From (2.11) we see

$$
\zeta r\left(z^{d}\right)=r(z)+\sum_{i \in J_{1}} c_{i} \frac{z^{m_{i}}}{z^{m_{i}}-1}-\sum_{i \in J_{2}} c_{i} \frac{z^{m_{i}}}{z^{m_{i}}+1} .
$$

With $\underline{c}$ and $r$ as above, we define the rational function $s$ by

$$
s(z):=r(z)-\sum_{i \in J_{1}} \sum_{\tau=0}^{t(i)-1} \frac{c_{i} z^{d^{\tau} k_{i}}}{\zeta^{t(i)-\tau}\left(z^{d^{\tau} k_{i}}-1\right)}+\sum_{i \in J_{2}} \sum_{\tau=0}^{t(i)-1} \frac{c_{i} z^{d^{\tau} k_{i}}}{\zeta^{t(i)-\tau}\left(z^{d^{\tau} k_{i}}+1\right)} .
$$

This new function satisfies

$$
\begin{gathered}
\zeta s\left(z^{d}\right)=s(z)+\sum_{i \in J_{1}} \frac{c_{i} z^{k_{i}}}{\zeta^{t(i)}\left(z^{k_{i}}-1\right)}-\sum_{i \in J_{2}} \frac{c_{i} z^{k_{i}}}{\zeta^{t(i)}\left(z^{k_{i}}+1\right)} \\
=s(z)+\sum_{i \in J_{1}} \frac{c_{i}}{\zeta^{t(i)}}-\sum_{i \in J_{2}} \frac{c_{i}}{\zeta^{t(i)}}+\sum_{i \in J_{1}} \frac{c_{i}}{\zeta^{t(i)}\left(z^{k_{i}}-1\right)}+\sum_{i \in J_{2}} \frac{c_{i}}{\zeta^{t(i)}\left(z^{k_{i}}+1\right)} .
\end{gathered}
$$

Since all polynomials $z^{k_{i}}-1, z^{k_{i}}+1(i=1, \ldots, m)$ divide $z^{L}-1$, where $L:=2 \operatorname{lcm}\left(k_{1}, \ldots, k_{m}\right)$, it follows from [Nis97, Lemma 1] that $s$ must be of the form

$$
s(z)=\frac{a(z)}{z^{L}-1}
$$

with some $a \in \mathbb{C}[z]$. By considering poles on the right-hand side of the first line of (2.12), one easily concludes $a \neq 0$. Moreover, considering the same line near $\infty$, we obtain $\operatorname{deg} a \leq L$, whence $\sigma:=s(\infty) \in \mathbb{C}$ and $s_{1}(z):=s(z)-\sigma$ tends to 0 as $z \rightarrow \infty$. All in all, we conclude from the second line of $(2.12)$

$$
\begin{aligned}
\zeta s_{1}\left(z^{d}\right) & =s_{1}(z)+\sum_{i \in J_{1}} \frac{c_{i}}{\zeta^{t(i)}\left(z^{k_{i}}-1\right)}+\sum_{i \in J_{2}} \frac{c_{i}}{\zeta^{t(i)}\left(z^{k_{i}}+1\right)} \\
& =s_{1}(z)+\sum_{i=1}^{m} \frac{c_{i}}{\zeta^{t(i)}\left(z^{k_{i}}-1\right)}-\sum_{i \in J_{2}} \frac{2 c_{i}}{\zeta^{t(i)}\left(z^{2 k_{i}}-1\right)} .
\end{aligned}
$$

Let now $d \mid 2 k_{i}$ exactly for $i \in\{i(1), \ldots, i(p)\}$ ( $i \in J_{2}$, clearly such $i$ can exist only if $d$ is even), and write $2 k_{i}=d \ell_{i}$ for these $i$. Then $d \nmid \ell_{i}$ and the $\ell_{i}$ 's are distinct. Assume that $j(1), \ldots, j(q)$ are those values $i \in J_{2}$ with $d \nmid 2 k_{i}$. Since an equation $\ell_{i(u)}=2 k_{j(v)}$ leads to the contradiction $d \mid k_{i(u)}$, the intersection $\left\{\ell_{i(1)}, \ldots, \ell_{i(p)}\right\} \cap\left\{2 k_{j(1)}, \ldots, 2 k_{j(q)}\right\}$ is empty. It follows that the rational function

$$
S(z):=s_{1}(z)+\sum_{u=1}^{p} \frac{2 c_{i(u)}}{\zeta^{t(i(u))+1}\left(z^{\ell_{i(u)}}-1\right)}
$$


satisfies

$$
\zeta S\left(z^{d}\right)-S(z)=\sum_{i=1}^{m} \frac{c_{i}}{\zeta^{t(i)}\left(z^{k_{i}}-1\right)}-\sum_{u=1}^{p} \frac{2 c_{i(u)}}{\zeta^{t(i(u))+1}\left(z^{\ell_{i(u)}}-1\right)}-\sum_{v=1}^{q} \frac{2 c_{j(v)}}{\zeta^{t(j(v))}\left(z^{2 k_{j(v)}}-1\right)},
$$

where, without loss of generality, we may assume $\ell_{i(1)}>\cdots>\ell_{i(p)}$ and $2 k_{j(1)}>\cdots>2 k_{j(q)}$.

The possible poles on both sides of (2.13) are roots of unity. If there ever is a pole, let a primitive $N$ th root of unity be one of these on the left-hand side with maximal $N$. Crucial to our final reasoning will be the fact $d \mid N$.

Indeed, this divisibility property follows from the proof of [BV15c, Lemma 3.3], but, for the sake of completeness, we briefly explain here the reasoning. For this purpose, we may write down the partial fraction decomposition of $S$ as

$$
S(z)=\sum_{\delta \mid L} s_{\delta}(z) \text { with } s_{\delta}(z):=\sum_{\substack{j=0 \\(j, \delta)=1}}^{\delta-1} \frac{s_{\delta, j}}{z-\zeta_{\delta}^{j}}
$$

where the $s_{\delta, j}$ 's are complex constants and $\zeta_{\delta}:=e^{2 \pi i / \delta}$.

From this definition of $s_{\delta}$ for positive divisors $\delta$ of $L$ we obtain

$$
s_{\delta}\left(z^{d}\right)=\sum_{\substack{j=0 \\(j, \delta)=1}}^{\delta-1} \frac{s_{\delta, j}}{z^{d}-\zeta_{\delta}^{j}}=\sum_{\substack{j=0 \\(j, \delta)=1}}^{\delta-1} s_{\delta, j} \sum_{\kappa=0}^{d-1} \frac{1}{d \zeta_{d \delta}^{(j+\kappa \delta)(d-1)}\left(z-\zeta_{d \delta}^{j+\kappa \delta}\right)} .
$$

Suppose, from now on, $p_{1}^{\nu(1)} \cdot \ldots \cdot p_{\omega}^{\nu(\omega)}$ to be the canonical factorization of $d$. Assume that $p_{1}, \ldots, p_{\sigma}$ are not divisors of $\delta$ but $p_{\sigma+1}, \ldots, p_{\omega}$ are, where we have to consider the cases $\sigma=0, \ldots, \omega$. Then we have the following equivalence

$$
(j, \delta)=1 \Longleftrightarrow(j+\kappa \delta, \delta)=\left(j+\kappa \delta, d \delta / \prod_{i=1}^{\sigma} p_{i}^{\nu(i)}\right)=1
$$

with the usual convention here and later that empty products (or sums) equal 1 (or 0 , respectively). Now, any positive divisor $D$ of $p_{1} \cdot \ldots \cdot p_{\sigma}$ is relatively prime to $\delta$, whence there are precisely $\frac{d}{D}$ numbers $\kappa \in\{0, \ldots, d-1\}$ satisfying $D \mid(j+\kappa \delta)$. Thus, by the well-known inclusion-exclusion principle, we can say that, for fixed coprime $j, \delta$, the number of $\kappa \in\{0, \ldots, d-1\}$ such that $j+\kappa \delta$ is prime to $p_{1} \cdot \ldots \cdot p_{\sigma}$ (or equivalently to $\prod_{i=1}^{\sigma} p_{i}^{\nu(i)}$ ) equals $d \prod_{i=1}^{\sigma}\left(1-1 / p_{i}\right)$. Therefore we can note that, for fixed coprime $j, \delta$, there are exactly $d \prod_{i=1}^{\sigma}\left(1-1 / p_{i}\right)$ values $\kappa \in\{0, \ldots, d-1\}$ such that $(j+\kappa \delta, d \delta)=1$ holds. Hence we conclude

$$
s_{\delta}\left(z^{d}\right)=\sum_{\substack{j=0 \\(j, d \delta)=1}}^{d \delta-1} \frac{s_{\delta, j-[j / \delta] \delta}}{d \zeta_{d \delta}^{j(d-1)}\left(z-\zeta_{d \delta}^{j}\right)}+\Sigma_{\delta}(z)
$$

from the double sum in (2.14). The rational function $\Sigma_{\delta}$ in (2.15) vanishes identically in case $\sigma=0$, whereas, in the cases $1 \leq \sigma \leq \omega$, it may have poles at certain primitive $\rho$ th roots of unity but with $\rho<d \delta$ only. Since $s_{\delta} \neq 0$ is equivalent to the fact that not all $s_{\delta, j-[j / \delta] \delta}, j \in\{0, \ldots, d \delta-1\}$ and prime to $d \delta$, vanish, we conclude from (2.15) that, in this case of $\delta$, the difference $s_{\delta}\left(z^{d}\right)-s_{\delta}(z)$ has poles at $(d \delta)$ th roots of unity. Thus, the number $N$ defined after (2.13) must be of the form $d \delta$, whence $d \mid N$ holds.

If the set $J_{2}$ is empty $(p+q=0)$, then we obtain $N=k_{1}$ from the right-hand side of (2.13), hence $d \mid k_{1}$, a contradiction, and it suffices to subsequently consider only the case $p+q \geq 1$.

In case $d=2$, we have $q=0$ and $k_{i}=\ell_{i}$ for all $i \in J_{2}$. Thus, the right-hand side of (2.13) is of the form

$$
\sum_{i=1}^{m} \frac{c_{i}}{\zeta^{t(i)}\left(z^{k_{i}}-1\right)}-\sum_{i \in J_{2}} \frac{2 c_{i}}{\zeta^{t(i)+1}\left(z^{k_{i}}-1\right)}
$$


whence again $N=k_{1}$, a contradiction.

We next suppose $d \geq 3$. If $k_{1} \neq \max \left\{\ell_{i(1)}, \ldots, \ell_{i(p)}, 2 k_{j(1)}, \ldots, 2 k_{j(q)}\right\}$, we are led to the same contradiction as before. Hence, let $k_{1}$ be equal to that maximum. This implies $k_{1}=2 k_{j(1)}$ observing that $\ell_{i(1)}<k_{1}$ follows from $d \geq 3$. If $c_{1} / \zeta^{t(1)} \neq 2 c_{j(1)} / \zeta^{t(j(1))}$, we have our contradiction, whence $c_{1}=2 c_{j(1)} \zeta^{t(1)-t(j(1))}$ must hold. We may now continue in the same way and obtain a contradiction unless $\left\{k_{1}, \ldots, k_{p+q}\right\}=\left\{\ell_{i(1)}, \ldots, \ell_{i(p)}, 2 k_{j(1)}, \ldots, 2 k_{j(q)}\right\}$. Moreover, for each $i \in\{1, \ldots, p+q\}$, there must exist a unique $\mu(i) \in\{i(1), \ldots, i(p), j(1), \ldots, j(q)\}$ such that $c_{i}=2 c_{\mu(i)} \zeta^{t(i)-t(\mu(i))-\delta}$ with $\delta=1$ if $\mu(i) \in\{i(1), \ldots, i(p)\}$ and $\delta=0$ otherwise. If $p+q<m$, then $N=k_{p+q+1}$ holds under the preceding conditions, and we end up at our 'standard' contradiction. Therefore, the only remaining possibility is that $p+q=m, J_{1}=\emptyset$. In this case, the above conditions lead to $c_{1}=2^{j} \zeta^{\nu} c_{1}$ with some $j \in\{1, \ldots, m\}, \nu \in \mathbb{Z}$, and this contradiction completes the proof of Lemma 2.

We next apply some ideas introduced in [Nis02],[NTT99] (see also [BV14],[BV15b]) to the functions

$$
g_{i, d}(z):=\mathcal{G}_{d}\left(z^{m_{i}}\right), \quad f_{i, d}(z):=\mathcal{F}_{d}\left(z^{m_{i}}\right) \quad(i=1, \ldots, n),
$$

the $\mathcal{G}_{d}, \mathcal{F}_{d}$ as defined in (1.4). We obtain from Lemma 2 and [BV14, Lemma 5] the following

Lemma 3. Under the assumptions of Lemma 2, the functions

$$
g_{i, d^{j}}(z)=\sum_{h=0}^{\infty} \frac{a_{h} z^{m_{i} d^{j h}}}{1-z^{m_{i} d^{j h}}}\left(i \in I_{1}, j \in \mathbb{N}\right), \quad f_{i, d^{j}}(z)=\sum_{h=0}^{\infty} \frac{a_{h} z^{m_{i} d^{j h}}}{1+z^{m_{i} d^{j h}}}\left(i \in I_{2}, j \in \mathbb{N}\right)
$$

are algebraically independent over $\mathbb{C}(z)$.

Similarly to the proof of [BV14, Theorem 3], and noting that condition (1.5) implies (1.3), this lemma leads to the following result.

Theorem 6. Let $m_{1}, \ldots, m_{n}$ be $n \geq 2$ positive integers satisfying (1.5), and let $\alpha \in \overline{\mathbb{Q}}^{\times} \cap \mathbb{D}$. Then the numbers $\mathcal{G}_{r}\left(\alpha_{i}\right)$ with $i \in I_{1}, r \in \mathbb{N} \backslash\{1\}$, and $\mathcal{F}_{r}\left(\alpha_{i}\right)$ with $i \in I_{2}, r \in \mathbb{N} \backslash\{1\}$ are algebraically independent. In particular, the numbers $G_{r}\left(\alpha_{i}\right)\left(i \in I_{1}, r \in \mathbb{N} \backslash\{1\}\right), F_{r}\left(\alpha_{i}\right)\left(i \in I_{2}, r \in \mathbb{N} \backslash\{1\}\right)$ are algebraically independent.

\section{Proof of Theorems 2 and 3}

To this end, we denote $I_{1}:=\left\{i: \alpha_{i}=\alpha^{m_{i}}\right\}, I_{2}:=\left\{i: \alpha_{i}=-\alpha^{m_{i}}\right\}$. Clearly, these $I_{j}$ satisfy the conditions of Lemma 2. Further, let

$$
h_{i}(\zeta, z):=G_{d}\left(\zeta, z^{m_{i}}\right) \text { for } i \in I_{1}, \quad h_{i}(\zeta, z):=G_{d}\left(\zeta,-z^{m_{i}}\right) \text { for } i \in I_{2},
$$

where $G_{d}(a, z)$ (and its $F$-analogue) is defined in (2.9). If $i \in I_{2}$, then $h_{i}(\zeta, z)=-F_{d}\left(\zeta, z^{m_{i}}\right)$ for odd $d$, and $h_{i}(\zeta, z)=G_{d}\left(\zeta, z^{m_{i}}\right)+2 z^{m_{i}} /\left(z^{2 m_{i}}-1\right)$ for even $d$. Therefore, Lemma 2 immediately gives

Lemma 4. Let $m_{1}, \ldots, m_{n}$ be $n \geq 2$ positive integers satisfying (1.3). Then, for any root of unity $\zeta$, the functions $h_{i}(\zeta, z)(i=1, \ldots, n)$ are linearly independent over $\mathbb{C}$ modulo $\mathbb{C}(z)$.

By using [Nis96, Theorem 3.3.11], this lemma provides us directly Theorem 2. 
Lemma 4 leads also to an analogue of Lemma 3 for the functions

$$
h_{i, d}(z):=g_{i, d}(z) \text { if } i \in I_{1}, \quad h_{i, d}(z):=\sum_{h=0}^{\infty} \frac{a_{h}\left(-z^{m_{i}}\right)^{d^{h}}}{1-\left(-z^{m_{i}}\right)^{d^{h}}} \text { if } i \in I_{2} .
$$

Lemma 5. If the assumptions of Lemma 4 are satisfied, then the functions $h_{i, d^{j}}(z)(i=1, \ldots, n, j \in$ $\mathbb{N})$ are algebraically independent over $\mathbb{C}(z)$.

Analogously to Theorem 6, this lemma implies Theorem 3.

\section{Proof of Theorems 4 and 5}

In the following, we want to study algebraic independence of the functions $G_{d}$ and $F_{d}$. By (1.6), it is natural to suppose $d \geq 3$ for this consideration. First we note that, for any $m \in \mathbb{N}$, the functional equation

$$
\zeta r\left(z^{d}\right)=r(z)+\frac{z^{m}}{z^{m}-1}-\frac{2 z^{2 m}}{z^{2 m}-1}+\frac{z^{m}}{z^{m}+1} .
$$

has $r(z)=0$ as a solution. Thus, the functions $G_{d}\left(\zeta, z^{m}\right), G_{d}\left(\zeta, z^{2 m}\right)$, and $F_{d}\left(\zeta, z^{m}\right)$ are linearly dependent over $\mathbb{C}$. More generally, if

$$
\frac{m_{j}}{2 m_{i}} \in d^{\mathbb{Z}}
$$

holds, then the functions $g_{j}(z), g_{i}(z)$, and $f_{i}(z)$ introduced in (2.10) are linearly dependent over $\mathbb{C}$ modulo $\mathbb{C}(z)$. Indeed, if $m_{j}=d^{t} 2 m_{i}$ with $t \geq 0$, then the rational function

$$
r_{1}(z):=-2 \sum_{\tau=0}^{t-1} \frac{z^{d^{\tau} 2 m_{i}}}{\zeta^{t-\tau}\left(z^{d^{\tau} 2 m_{i}}-1\right)}
$$

satisfies

$$
\zeta r_{1}\left(z^{d}\right)=r_{1}(z)-\frac{2 z^{m_{j}}}{z^{m_{j}}-1}+\frac{z^{m_{i}}}{\zeta^{t}\left(z^{m_{i}}-1\right)}+\frac{z^{m_{i}}}{\zeta^{t}\left(z^{m_{i}}+1\right)}
$$

implying

$$
r_{1}(z)=-2 g_{j}(z)+\zeta^{-t} g_{i}(z)-\zeta^{-t} f_{i}(z)
$$

Furthermore, if $d^{t} m_{j}=2 m_{i}$ with $t>0$, then

$$
r_{2}(z):=2 \sum_{\tau=0}^{t-1} \frac{z^{d^{\tau} m_{j}}}{\zeta^{t-\tau}\left(z^{d^{\tau} m_{j}}-1\right)}
$$

is a solution of

$$
\zeta r_{2}\left(z^{d}\right)=r_{2}(z)-2 \zeta^{-t} \frac{z^{m_{j}}}{z^{m_{j}}-1}+\frac{z^{m_{i}}}{z^{m_{i}}-1}+\frac{z^{m_{i}}}{z^{m_{i}}+1}
$$

implying

$$
r_{2}(z)=-2 \zeta^{-t} g_{j}(z)+g_{i}(z)-f_{i}(z) .
$$

This makes it evident that we have to suppose condition (1.7) for our linear independence considerations.

Lemma 6. Let $m_{1}, \ldots, m_{n}$ be $n \geq 2$ positive integers, let $d \geq 3$, and assume that conditions (1.3) and (1.7) are satisfied. Then, for any root of unity $\zeta$, the functions $G_{d}\left(\zeta, z^{m_{1}}\right), \ldots, G_{d}\left(\zeta, z^{m_{n}}\right), F_{d}\left(\zeta, z^{m_{1}}\right)$, 


\section{$\ldots, F_{d}\left(\zeta, z^{m_{n}}\right)$ are linearly independent over $\mathbb{C}$ modulo $\mathbb{C}(z)$.}

Proof. Assume, contrary to Lemma 6 , that there exists a $\underline{C}:=\left(b_{1}, \ldots, b_{n}, c_{1}, \ldots, c_{n}\right) \in \mathbb{C}^{2 n} \backslash\{\underline{0}\}$ such that $r(z):=\sum_{i=1}^{n}\left(b_{i} g_{i}(z)+c_{i} f_{i}(z)\right)$ is a rational function satisfying

$$
\zeta r\left(z^{d}\right)=r(z)+\sum_{i=1}^{n} b_{i} \frac{z^{m_{i}}}{z^{m_{i}}-1}-\sum_{i=1}^{n} c_{i} \frac{z^{m_{i}}}{z^{m_{i}}+1} .
$$

We may now argue as in the proof of Lemma 2 to obtain

$$
\zeta S\left(z^{d}\right)-S(z)=\sum_{i=1}^{n} \frac{b_{i}+c_{i}}{\zeta^{t(i)}\left(z^{k_{i}}-1\right)}-\sum_{u=1}^{p} \frac{2 c_{i(u)}}{\zeta^{t(i(u))+1}\left(z^{\ell_{i(u)}}-1\right)}-\sum_{v=1}^{q} \frac{2 c_{j(v)}}{\zeta^{t(j(v))}\left(z^{2 k_{j(v)}}-1\right)}
$$

as an analogue of (2.13), where $p+q=n$ and some of $b_{i}, c_{i}$ may vanish. An equation $k_{i}=2 k_{j(v)}$ is impossible, by (1.7). Thus, $k_{i} \neq 2 k_{j(v)}$ for all pairs $(i, j(v))$, and similarly $k_{i} \neq \ell_{i(u)}$ for all pairs $(i, i(u))$. Further, $\left\{\ell_{i(1)}, \ldots, \ell_{i(p)}\right\} \cap\left\{2 k_{j(1)}, \ldots, 2 k_{j(q)}\right\}=\emptyset$ was shown in the proof of Lemma 2. All in all, this means that $\left\{k_{1}, \ldots, k_{n}, \ell_{i(1)}, \ldots, \ell_{i(p)}, 2 k_{j(1)}, \ldots, 2 k_{j(q)}\right\}$ is a set of $2 n$ distinct positive integers, each one not divisible by $d$. Moreover, condition $\underline{C} \neq \underline{0}$ implies $\left(b_{1}+c_{1}, \ldots, b_{n}+c_{n}, c_{1}, \ldots, c_{n}\right) \neq \underline{0}$. Therefore, if we now define $N$ for the equation (4.16) as we did it for (2.13), then we get $d \mid N$ from the left-hand side of (4.16), but clearly $d \nmid N$ from its right-hand side, a contradiction.

To establish Theorem 4, we proceed similarly to our proof of Theorem 2 in the previous section. Moreover, the following analogue of Lemma 5 holds.

Lemma 7. Let $m_{1}, \ldots, m_{n}$ be $n \geq 2$ positive integers, let $d \geq 3$, and assume that conditions (1.3) and (1.7) are satisfied. Then the functions $g_{i, d^{j}}(z), f_{i, d^{j}}(z)(i=1, \ldots, n ; j \in \mathbb{N})$ are algebraically independent over $\mathbb{C}(z)$.

Outline of Proof. By noting that (1.8) implies (1.3) and (1.7) for any $d \geq 3$, the proof of Theorem 5 runs very much parallel to that of Theorem 6 .

Acknowledgement. The authors are grateful to the referees for suggestions improving the work.

\section{References}

[BV15a] P. Bundschuh and K. Väänänen, Algebraic independence of certain Mahler functions and of their values, J. Aust. Math. Soc. 98 (2015) 289-310.

[BV14] P. Bundschuh and K. Väänänen, Algebraic independence of reciprocal sums of certain Fibonacci-type numbers, arXiv:1403.5510v1 [math. NT] (2014).

[BV15b] P. Bundschuh and K. Väänänen, Algebraic independence of reciprocal sums of powers of certain Fibonacci-type numbers, Funct. Approx. Comment. Math. (to appear).

[BV15c] P. Bundschuh and K. Väänänen, Guided by Schwarz' functions: a walk through the garden of Mahler's transcendence method, submitted.

[Coo12] M. Coons, Extension of some theorems of W. Schwarz, Canad. Math. Bull. 55 (2012) 60-66.

[Coo13] M. Coons, On the rational approximation of the sum of the reciprocals of the Fermat numbers, The Ramanujan Journal 30 no. 1 (2013) 39-65.

[Mah69] K. Mahler, Remarks on a paper by W. Schwarz, J. Number Theory 1 (1969) 512-521.

[Nis96] K. Nishioka, Mahler Functions and Transcendence LNM 1631 (Springer, Berlin et al., 1996).

[Nis97] K. Nishioka, Algebraic independence of reciprocal sums of binary recurrences, Monatsh. Math. 123 (1997) 135-148.

[Nis02] K. Nishioka, Algebraic independence of reciprocal sums of binary recurrences II, Monatsh. Math. 136 (2002) 123-141.

[NTT99] K. Nishioka, T. Tanaka, and T. Toshimitsu, Algebraic independence of sums of reciprocals of the Fibonacci numbers, Math. Nachr. 202 (1999) 97-108. 
[Sch67] W. Schwarz, Remarks on the irrationality and transcendence of certain series, Math. Scand. 20 (1967) $269-274$.

[Tan05] T. Tanaka, Algebraic independence of the values of power series, Lambert series, and infinite products generated by linear recurrences, Osaka J. Math. 42 (2005) 487-497.

Peter Bundschuh

Mathematisches Institut

Universität zu Köln

Weyertal 86-90

50931 Köln, Germany

e-mail:pb@math.uni-koeln.de
Keijo Väänänen

Department of Mathematical Sciences

University of Oulu

P. O. Box 3000

90014 Oulu, Finland

e-mail: keijo.vaananen@.oulu.fi 